\title{
The Hot Big Bang and Beyond
}

\author{
Michael S. Turner \\ Departments of Physics and of Astronomy \& Astrophysics \\ Enrico Fermi Institute, The University of Chicago, Chicago, IL 60637-1433 \\ NASA/Fermilab Astrophysics Center \\ Fermi National Accelerator Laboratory, Batavia, IL 60510-0500
}

\begin{abstract}
The hot big-bang cosmology provides a reliable accounting of the Universe from about $10^{-2} \mathrm{sec}$ after the bang until the present, as well as a robust framework for speculating back to times as early as $10^{-43} \mathrm{sec}$. Cosmology faces a number of important challenges; foremost among them are determining the quantity and composition of matter in the Universe and developing a detailed and coherent picture of how structure (galaxies, clusters of galaxies, superclusters, voids, great walls, and so on) developed. At present there is a working hypothesis - cold dark matter-which is based upon inflation and which, if correct, would extend the big bang model back to $10^{-32} \mathrm{sec}$ and cast important light on the unification of the forces. Many experiments and observations, from CBR anisotropy experiments to Hubble Space Telescope observations to experiments at Fermilab and CERN, are now putting the cold dark matter theory to the test. At present it appears that the theory is viable only if the Hubble constant is smaller than current measurements indicate (around $30 \mathrm{~km} \mathrm{~s}^{-1} \mathrm{Mpc}^{-1}$ ), or if the theory is modified slightly, e.g., by the addition of a cosmological constant, a small admixture of hot dark matter ( $5 \mathrm{eV}$ "worth of neutrinos"), more relativistic particles, or a tilted spectrum of density perturbations.
\end{abstract}




\section{Successes}

The success of the hot big-bang cosmology (or standard cosmology as it is known) is simple to describe: It provides a reliable and tested accounting of the Universe from a fraction of a second after the bang (temperatures of order a few $\mathrm{MeV}$ ) until the present 15 Billion years later (temperature $2.726 \mathrm{~K}$ ). When supplemented by the standard model of particle physics and various ideas about physics at higher energies (e.g., supersymmetry, grand unification, and superstrings) it provides a sound foundation for speculations about the Universe back to $10^{-43} \mathrm{sec}$ after the bang (temperatures of $10^{19} \mathrm{GeV}$ ) and perhaps even earlier [1].

The fundamental observational data that support the standard cosmology are: the universal expansion (Hubble flow of galaxies); the cosmic background radiation (CBR); and the abundance of the light elements $\mathrm{D},{ }^{3} \mathrm{He},{ }^{4} \mathrm{He}$, and ${ }^{7} \mathrm{Li}$. The Hubble law $\left(z \simeq v / c \simeq H_{0} d\right)$ has been tested to a redshift $z \sim 0.05$ [2] and the highest redshift object is a QSO with $z=4.90$. (One plus redshift is the size of the Universe today relative to its size at the time of emission, $1+z=R_{0} / R_{E} ; \mathrm{R}$ is the cosmic scale factor, or relative size of the Universe).

The surface of last scattering for the CBR is the Universe at an age of a few hundred thousand years $(T \sim 0.3 \mathrm{eV}$ and redshift $z \sim 1100)$. COBE has determined its temperature to be $2.726 \pm 0.005 \mathrm{~K}$ and constrains any deviations from a black-body spectrum to be less than $0.03 \%$ [3]. The CBR temperature is very uniform: the difference between two points separated by angles from arcminutes to $90^{\circ}$ is less than $300 \mu K$, indicating that the Universe had a very smooth beginning. There is a dipole anisotropy in the CBR temperature of about $3 \mathrm{mK}$, due to our motion with respect to the cosmic rest frame (the "peculiar velocity" of the Local Group is $620 \mathrm{~km} \mathrm{~s}^{-1}$ toward the constellation Leo), and temperature differences on angular scales from $0.5^{\circ}$ to $90^{\circ}$ have been detected by about ten experiments at the level of about $30 \mu \mathrm{K}[4]$.

The abundance of the light elements, which range from about $24 \%$ for ${ }^{4} \mathrm{He}$ to $10^{-5}$ for $\mathrm{D}$ and ${ }^{3} \mathrm{He}$ and $10^{-10}$ for ${ }^{7} \mathrm{Li}$ are consistent with the predictions of big-bang nucleosynthesis. The synthesis of the light elements occurred when the Universe was of order seconds old and the temperature was of order $\mathrm{MeV}$. Big-bang nucleosynthesis is the earliest test of the standard cosmology, and it passes it with flying colors [5].

Finally, the standard cosmology provides a general framework for un- 
derstanding how the Universe evolved from a very smooth beginning to the abundance of structure observed today-galaxies, clusters of galaxies, superclusters, voids, great walls and so on. Small (primeval) variations in the matter density $\left(\delta \rho / \rho \sim 10^{-5}\right)$ were amplified by gravity (the Jeans' instability in the expanding Universe) eventually resulting in the structure seen today [6]. The CBR temperature fluctuations detected on angular scales from $0.5^{\circ}$ to $90^{\circ}$ are strong evidence for the existence of primeval density fluctuations.

In addition to accounting for the evolution of the Universe from $0.01 \mathrm{sec}$ onward, the standard cosmology provides a sound framework for speculating about even earlier times-at least back to the Planck time $\left(10^{-43}\right.$ sec and temperature of order $10^{19} \mathrm{GeV}$ ). Of course, advances in particle theory have played an important role here.

According to the standard model of particle physics the fundamental particles are point-like quarks and leptons whose interactions are weak enough to treat perturbatively. The cosmological implications of this are profound: The Universe at temperatures greater than about $150 \mathrm{MeV}$ (times earlier than $10^{-5} \mathrm{sec}$ ) consisted of a hot, dilute gas of quarks, leptons, and gauge bosons (photons, gluons, and at high enough temperatures $W$ and $Z$ bosons, the carriers of the electromagnetic, strong and weak forces). The standard model of particle physics, which has been tested up to energies of several hundred $\mathrm{GeV}$, provides the microphysics needed to discuss times as early as $10^{-11} \mathrm{sec}$. In addition, it provides a firm platform for speculations about the unification of forces and particles (e.g., supersymmetry and grand unification), and in turn, the necessary microphysics for extending cosmological speculations back to the Planck epoch. Earlier than the Planck time a quantum description of gravity is needed, and superstring theory is a good candidate for such.

While the hot big-bang cosmology and modern particle theory allow "sensible" - and very interesting - speculations about the early Universe, there is no evidence yet that any of these speculations is correct. However, contrast this with the situation before the early 1970s. The count of "elementary particles" (baryons and mesons) had exceeded 100 and was growing exponentially with mass; this, the strength of their interactions and their finite sizes precluded any sensible speculation about the Universe at times earlier than about $10^{-5}$ sec [7]. 


\section{Challenges}

Cosmology is not without its challenges. In its success, the hot big-bang model has allowed cosmologists to ask even deeper questions. They include: What is the quantity and composition of the ubiquitous dark matter in the Universe? What is the origin and nature of the primeval density perturbations that seeded structure and precisely how did the structure form? What is the origin of the cosmic asymmetry between matter and antimatter? Why is the observed portion of the Universe so smooth and flat? And does this mean that the entire Universe is the same? Are there observational consequences of the phase transitions that the Universe has undergone (transition from quarks to nucleons and related particles, electroweak symmetry breaking, and possibly others) during its earliest moments? Are there observable consequences of the quantum-gravity epoch? Why does the Universe have four dimensions? What caused the expansion in the first place?

The first two of these challenges, the nature of the dark matter and the details of structure formation, are in my opinion the most pressing and may well be resolved soon. Thus they offer an excellent opportunity for extending the big-bang cosmology back to much earlier times.

That is not to say that the other challenges are not important or do not have potential for advancing our understanding. In addition, there more practical challenges that I have not listed; for example, a precise determination of the three traditional parameters used to describe "our world model," the Hubble constant, the deceleration parameter, and the cosmological constant, or an explanation for the primeval magnetic fields required to seed the magnetic fields seen throughout the Universe today.

\subsection{Discard the big bang?}

There are few who believe the big bang faces challenges of such enormity that they will led to its downfall [8]. For example, if the Hubble constant is as large as some determinations indicate, say around $80 \mathrm{~km} \mathrm{~s}^{-1} \mathrm{Mpc}^{-1}$ and the oldest stars are as old as some determinations indicate, say around $16 \mathrm{Gyr}$, then a real dilemma exists because without recourse to a cosmological constant the time back to the back is less than 12 Gyr [9].

Before the COBE discovery of CBR anisotropy in 1992 [10], some argued that the absence of anisotropy precluded inhomogeneity of a size large enough 
to seed all the structure seen today. The big-bang has weathered that storm: Fluctuations in the CBR temperature have been detected and are now seen on scales from $0.5^{\circ}$ to $90^{\circ}$. In fact, careful calculations indicate that if anything the level of temperature fluctuations seen is slightly larger than is expected, given the structure seen today $[4,11]$.

The only two competitors to the big bang are the quasi steady-state model [12] and the plasma universe model. At the moment the problems that these models face seem far more daunting: themalization of starlight to produce $2.726 \mathrm{~K}$ black body background with no spectral distortion (quasi steady-state) and the formulation of a model definite enough to be tested (plasma universe). Until these models (or another model) can account for the cosmological data that have been firmly established (expansion, CBR, light elements, and structure formation), the standard cosmology is without a serious competitor.

\subsection{Dark Matters}

An accurate inventory of matter in the Universe still eludes cosmologists. What we do know is: (i) luminous matter (i.e., matter closely associated with bright stars) contributes a fraction of the critical density that is about $0.003 h^{-1}$ [13]; (ii) based upon big-bang nucleosynthesis baryons contributions a fraction of critical density between $0.009 h^{-2}$ and $0.022 h^{-2}$ [5], which for a generous range of the Hubble constant corresponds to between about 0.01 and 0.15 of the critical density; (iii) there are indications that the fraction of critical density contributed by all forms of matter is at least $0.1-0.3[14]$ - flat rotation curves of spiral galaxies, virial mass determinations of rich clusters-and perhaps around the critical density-the peculiar motions of galaxies, cluster mass determinations based upon gravitational lensing and x-ray measurements $[14,15]$. (Here the Hubble constant $H_{0}=100 h \mathrm{~km} \mathrm{~s}^{-1} \mathrm{Mpc}^{-1}$ and the critical density $\rho_{\text {crit }}=3 H_{0}^{2} / 8 \pi G=$ $1.88 h^{2} \times 10^{-29} \mathrm{~g} \mathrm{~cm}^{-3} \simeq 1.05 h^{2} \times 10^{4} \mathrm{eV} \mathrm{cm}^{-3}$.)

From this one concludes that: (i) most of the matter in the Universe is dark; (ii) most of the baryons are dark; (iii) the dark matter is not closely associated with bright stars, i.e., it is more diffusely distributed, e.g., in the extended halos of spiral galaxies; and (iv) if the total mass density is greater than about $20 \%$ of the critical density, then there must be another form of matter since baryons can at most account for $15 \%$ of the critical density (and 
only for a low value of the Hubble constant).

The case for $\Omega_{0} \gtrsim 0.2$ and nonbaryonic dark matter receives additional support, albeit indirectly, from other lines of reasoning. First, it is difficult to reconcile all the data concerning the formation of structure in the Universe with a theory that has no nonbaryonic dark matter (the one model that may be able to do so is Peebles' primeval baryon isocurvature model or PBI [16]). Second, the most compelling and comprehensive theory of the early Universe, inflation [17], predicts a flat Universe (total energy density equal to the critical density) and thus requires something other than baryons. Third, since the deviation of $\Omega$ from unity grows with time, if $\Omega_{0}$ is not equal to unity, the epoch when $\Omega_{0}$ just begins to deviate significantly from one is a special epoch and is today(!) (this is often called the Dicke-Peebles timing argument).

Last but not least, there are three compelling candidates for the nonbaryonic dark matter: an axion of mass between $10^{-6} \mathrm{eV}$ and $10^{-4} \mathrm{eV}$; a neutralino of mass between $10 \mathrm{GeV}$ and $1000 \mathrm{GeV}$; and a light neutrino species of mass between $10 \mathrm{eV}$ and about $50 \mathrm{eV}$ [18]. By compelling, I mean these particles arose out of efforts to unify the forces of Nature, and the fact that a particle was predicted whose relic mass density is close to critical is a bonus. This may be the "Grand Hint" or the "Great Misdirection."

For the axion, the underlying particle physics is Peccei-Quinn symmetry which is the most attractive solution to the so-called strong-CP problem (the fact that standard model of particle physics predicts the electric dipole moment of the neutron to be almost ten orders of magnitude larger than the current upper limit). For the neutralino, it is supersymmetry, the symmetry that relates fermions and bosons and which helps to explain the large discrepancy between the weak scale $(300 \mathrm{GeV})$ and the Planck scale and may hold the key to unifying gravity with the other forces. Unlike the axion or the neutralino, neutrinos are known to exist, come in three varieties, and have a relic abundance known to three significant figures $\left(113 \mathrm{~cm}^{-3}\right.$ per species $)$; the only issue is their mass. Almost all attempts to unify the forces and particles of nature lead to the prediction that neutrinos have mass, often in the "eV range" (meaning anywhere from $10^{-6} \mathrm{eV}$ or smaller to $\mathrm{keV}$ ).

The axion and neutralino are referred to as "cold dark matter" because they move very slowly (neutralinos because they are heavy and axions because they were produced in the early Universe with very small momenta). Neutrinos on the other hand are referred to as "hot dark matter" because 
they move rapidly (due to their small mass). The distinction between the two is crucial for structure formation: at early times neutrinos can "run out" of overdense regions and into underdense regions, damping density perturbations on scales smaller than those corresponding to superclusters. This means that in the absence of additional seed perturbations that don't involve neutrinos (e.g., cosmic string) the sequence of structure formation in a hot dark matter universe proceeds from the "top down:" objects like superclusters form first and then fragment into smaller objects (galaxies and the like). Because there is now much evidence that "small objects" (galaxies, quasars, neutral hydrogen clouds, and clusters) were ubiquitous at redshifts from 1 to 4 , and "large objects" are just forming today, hot dark matter (without additional perturbations) is not viable.

To end on a sober note, at present the data can neither prove nor disprove either: (i) $\Omega_{0}=\Omega_{B} \simeq 0.15$; (ii) $\Omega_{0}=1$ with $\Omega_{B} \sim 0.05$ and $\Omega_{\mathrm{CDM}} \sim 0.95$. (In the first case the Hubble constant must be near its lower extreme since the nucleosynthesis measurement of $\Omega_{B}=0.009 h^{-2}-0.022 h^{-2}$.) In any case, I will devote the rest of this paper to the second, more radical possibility.

\subsection{Coherent picture of structure formation}

Because the energy densities of matter (baryons + CDM?) and radiation (photons, light neutrinos, and at early times all the other particles in the thermal plasma) evolve differently, $R^{-3}$ for matter and $R^{-4}$ for radiation, the energy density in radiation exceeded that in matter earlier at early times, $t \lesssim t_{\mathrm{EQ}} \sim 10^{4} \mathrm{yr}\left(T \gtrsim T_{\mathrm{EQ}} \sim 5 \mathrm{eV}\right.$ and $\left.R \lesssim R_{\mathrm{EQ}} \sim 3 \times 10^{-5} R_{\text {today }}\right)$. Moreover, matter density perturbations do not grow during the radiationdominated era, and thus the formation of structure did not begin in earnest until the epoch of matter-radiation equality. After that, (linear) perturbations in the matter grow as the scale factor, for a total (linear) growth factor of around 30,000. This factor sets the characteristic amplitude of density perturbations, about $\mathrm{few} \times 10^{-5}$ (nonlinear structures have formed by the present) and thus the expected size of temperature fluctuations in the CBR (density perturbations lead to comparable sized fluctuations in the CBR temperature).

The detection of CBR anisotropy at the level of about $10^{-5}$ validates the gravitational instability picture of structure formation. This success should be viewed in the same way that the evidence for a large primeval mass frac- 
tion of ${ }^{4} \mathrm{He}$ validated the the basic idea of primordial nucleosynthesis in the late 1960s. From this early success, big-bang nucleosynthesis developed into a coherent and detailed explanation for the abundances of $\mathrm{D},{ }^{3} \mathrm{He},{ }^{4} \mathrm{He}$ and ${ }^{7} \mathrm{Li}$, and now provides the earliest test of the big bang, the most reliable determination of the baryon density, and an important probe of particle physics. It is not unreasonable to hope that a detailed and coherent picture of structure formation develop and will lead to similar advances in our understanding of the Universe.

The two crucial elements must underlay any detailed picture: specification of the quantity and composition of the dark matter and the nature of the density perturbations. With regard to the latter, what is wanted is a mathematical description of the spectrum of density perturbations. For example, the Fourier components $\delta_{k}$ of the density field and their statistical properties.

At present there are three viable theories: cold dark matter models; topological-defect models [19]; and the primeval baryon isocurvature model (PBI) [16]. The effort being brought to bear on this problem-both experimental and theoretical - is great, and I am confident that at least two of these models, if not all three (!), will be falsified soon. It is my view that only cold dark matter will survive the next cut, but of course others may hold a different opinion.

Topological defect models, where the seeds are cosmic string, monopoles or textures produced in an early Universe phase transition and the dark matter is either neutrinos (cosmic string) or cold dark matter (textures), seem to predict CBR anisotropy on the degree scale that is significantly less than that measured. In addition, when normalized to the COBE measurements of anisotropy, they require a high level of "bias;" bias refers to the discrepancy between the light and mass distributions, $b \simeq\left(\delta n_{\mathrm{GAL}} / n_{\mathrm{GAL}}\right) /(\delta \rho / \rho)$, which is generally believe to be of order $1-2$. Much of the difficulty in assessing the defect models is on the theoretical side; density perturbations are constantly being produced as the defect network evolves and thus cannot easily be described by Fourier components whose evolution is simple.

The basic philosophy behind the PBI model is to explain the formation of structure by using "what is here," rather then what early-Universe theorists (like myself) hope is here! The parameters for PBI are: $\Omega_{0}=\Omega_{B} \sim 0.2$ and $H_{0} \sim 70 \mathrm{~km} \mathrm{~s}^{-1} \mathrm{Mpc}^{-1}$. An arbitrary power-law spectrum of fluctuations in the local baryon number (cut off at small scales to avoid difficulties with 
primordial nucleosynthesis) is postulated and its parameters (slope and normalization) are determined by the data (CBR fluctuations and large-scale structure). PBI has some serious problems: the baryon density violates the nucleosynthesis bound by a wide margin $\left(\Omega_{B} h^{2} \sim 0.1 \gg 0.02\right.$; it is difficult to make PBI consistent with the measurements of CBR anisotropy [20]. To wit, Peebles has considered variations on the basic theme [21] (e.g., adding a cosmological constant, or even cold dark matter). At the very least PBI provides a useful model against which scenarios that postulate exotic dark matter can be compared; at best, it may represent our Universe.

\section{Inflation and Cold Dark Matter}

Inflation represents a bold attempt to extend the standard big-bang cosmology to times as early as $10^{-32} \mathrm{sec}$ and to resolve some of the most fundamental questions in cosmology. In particular, inflation addresses squarely both the dark matter and structure formation problems, as well as providing an explanation for the flatness and smoothness of the Universe. If successful, inflation would be a truly remarkable addition to the standard cosmology.

At present there is no standard model of inflation; however, there are many viable models, all based on well defined speculations about physics at energy scales of around $10^{14} \mathrm{GeV}$ and higher [22]. Inflation makes three robust predictions: (1) spatially flat Universe [23]; (2) nearly scale-invariant spectrum of density (scalar metric) perturbations [24]; (3) nearly scale-invariant spectrum of gravity waves (tensor metric perturbations) [25].

With regard to metric perturbations; they are imprinted during inflation, arising from quantum mechanical fluctuations excited on extremely small scales $\left(\lesssim 10^{-23} \mathrm{~cm}\right)$, which are stretched to astrophysical scales $\left(\gtrsim 10^{25} \mathrm{~cm}\right)$ by the tremendous growth in the scale factor during inflation $\left(\gtrsim 10^{25}\right)$. In almost all models of inflation the statistics of the perturbations are gaussian, and the Fourier power spectrum, $P(k) \equiv\left|\delta_{k}\right|^{2}$, completely specifies the statistical properties of the density field.

While the metric perturbations are predicted to nearly scale invariant, the small deviations that can occur encode much about the underlying inflationary model. Likewise, the amplitudes of the metric perturbations are model dependent and hold equally important information. (Scale-invariant density perturbations means fluctuations in the gravitational potential that are equal 
on all scales at early times; for the gravitational waves, scale invariant means that all gravity waves cross the horizon with equal amplitude.)

The first prediction means the total energy density (including matter, radiation, and the vacuum energy density associated with a cosmological constant) is equal to the critical density, that is $\Omega_{0}=1$. Coupled with our knowledge of the baryon density, this implies that the bulk of matter in the Universe (95\% or so) must be nonbaryonic. The two simplest possibilities are hot dark matter or cold dark matter. Structure formation with hot dark matter has been studied, and, sadly, does not work; thus we are led to to cold dark matter.

For cold dark matter there is no damping of perturbations on small scales, and structure is built from the "bottom up:" Clumps of dark matter and baryons continuously merge to form larger objects. "Typical galaxies" are formed at redshifts $z \sim 1-2$; "rare objects" such as quasars and radio galaxies can form earlier from regions where the density perturbations have larger than average amplitude. Clusters form in the very recent past (redshifts less than order unity), and superclusters are just forming today. Voids naturally arise as regions of space are evacuated to form objects [26].

\subsection{Almost, but is something missing?}

Broadly speaking, testing the cold dark matter scenario involves measuring the quantity, composition, and distribution of dark matter and determining the spectrum of density perturbations. I have already discussed the current state of our knowledge of dark matter. While a host of observations provide information about the primeval spectrum of density perturbations, measurements of the anisotropy of the CBR and mapping the distribution of matter today (as traced by bright galaxies) are perhaps most crucial. (For reference, perturbations on scales of about $1 \mathrm{Mpc}$ correspond to galactic sized perturbations, on $10 \mathrm{Mpc}$ to cluster size perturbations, on $30 \mathrm{Mpc}$ to the large voids, and $100 \mathrm{Mpc}$ to the great walls.)

CBR anisotropy probes the power spectrum on large scales. The CBR temperature difference measured on a given angular scale is related to the power spectrum on a given length scale: $\lambda \sim(\theta / \mathrm{deg}) 100 h^{-1} \mathrm{Mpc}$. Since the COBE detection, a host of ground-based and balloon-borne experiments have also detected CBR anisotropy, on scales from about $0.5^{\circ}$ to $90^{\circ}$, at the level of around $30 \mu \mathrm{K}\left(\delta T / T \sim 10^{-5}\right)$. The measurements are consistent with 
the predictions of cold dark matter, though there are still large statistical uncertainties as well as concerns about contamination by foreground sources [4]. There is a great deal of experimental activity (more than ten groups), and measurements in the near future should improve the present situation significantly. The CBR contains important information on angular scales down to about $0.1 \mathrm{deg}$ (anisotropy on smaller angular scales is washed out due to the finite thickness of the last scattering surface). A follow-on to COBE, being studied in both Europe and the US, and a variety of earthbased and balloon-based experiments should hopefully map CBR anisotropy on scales from $0.1 \mathrm{deg}$ to $90 \mathrm{deg}$ in the next decade.

The COBE detection of CBR anisotropy not only provided the first evidence for the existence of primeval density perturbations, but also an unambiguous way to normalize the spectrum of density perturbations: Given the shape of the power spectrum (for cold dark matter, approximately scale invariant) the COBE measurement (on a scale of around $10^{3} h^{-1} \mathrm{Mpc}$ ) ties down the spectrum on all scales. This leads to definite predictions that can be tested by other CBR measurements and observations of large-scale structure.

The comparison of predictions for structure formation with present-day observations of the distribution of galaxies is very important, but fraught with difficulties. Theory most accurately predicts "where the mass is" (in a statistical sense) and the observations determine where the light is. Redshift surveys probe present-day inhomogeneity on scales from around one $\mathrm{Mpc}$ to a few hundred Mpc, scales where the Universe is nonlinear $\left(\delta n_{\mathrm{GAL}} / n_{\mathrm{GAL}} \gtrsim 1\right.$ on scales $\lesssim 8 h^{-1} \mathrm{Mpc}$ ) and where astrophysical processes undoubtedly play an important role (e.g., star formation determines where and when "mass lights up," the explosive release of energy in supernovae can move matter around and influence subsequent star formation, and so on). The distance to a galaxy is determined through Hubble's law $\left(d=H_{0}^{-1} z\right)$ by measuring a redshift; peculiar velocities induced by the lumpy distribution of matter are significant and prevent a direct determination of the actual distance. There are the intrinsic limitations of the surveys themselves: they are flux not volume limited (brighter objects are seen to greater distances and vice versa) and relatively small (e.g., the CfA slices of the Universe survey contains only about $10^{4}$ galaxies and extends to a redshift of about $z \sim 0.03$ ). Last but not least are the numerical simulations which bridge theory and observation; they are limited dynamical range (about a factor of 100 in length scale) and in microphysics (in the largest simulations only gravity, and in others only a 
gross approximation to the effects of hydrodynamics/thermodynamics).

This being said, redshift surveys do provide an important probe of the power spectrum on small scales $(\lambda \sim 1-300 \mathrm{Mpc})$. Even with their limitations redshift surveys (as well as other data) indicate that while the simplest version of COBE-normalized cold dark matter is in broad agreement with the data, the shape of the power spectrum as well as its amplitude on small scales is not quite right $[11,27]$. At least three possibilities come to mind: (i) the comparison of numerical simulations and the observations is still too primitive to draw firm conclusions; (ii) cold dark matter has much, but not all, of the "truth;" or (iii) cold dark matter has been falsified.

For three reasons I believe that it is worthwhile exploring possibility (ii), namely that something needs to be added to cold dark matter. First, cold dark matter is such an attractive theory and part of a bold attempt to extend greatly the standard cosmology. Second, many observations seem to point to the same problem (e.g., the abundance of x-ray clusters and the cluster-cluster correlation function). Third, there are other reasons to believe that the Universe is more complicated than the simplest model of cold dark matter.

\subsection{Five cold dark matter models}

Somewhat arbitrarily, standard cold dark matter has come to mean: precisely scale-invariant density perturbations; baryons + CDM only; and Hubble constant of $50 \mathrm{~km} \mathrm{~s}^{-1} \mathrm{Mpc}^{-1}$ (to ensure a sufficiently aged Universe with a Hubble constant still within the range of observations). This is the vanilla or default model, which, when normalized to COBE has too much power on small scales and the wrong spectral shape on larger scales.

The spectrum of density perturbations today depends not only upon the primeval spectrum (and the normalization on large scales provided by COBE), but also upon the energy content of the Universe. While the fluctuations in the gravitational potential were initially approximately scale invariant, the fact that the Universe evolved from an early radiation-dominated phase to a matter-dominated phase imposes a characteristic scale on the spectrum of density perturbations seen today; that scale is determined by the energy content of the Universe (the characteristic scale $\lambda_{\mathrm{EQ}} \sim 10 h^{-1} \mathrm{Mpc}_{*}^{1 / 2} / \Omega_{\text {matter }}$ where $g_{*}$ counts the relativistic degrees of freedom and $\Omega_{\text {matter }}=\Omega_{B}+\Omega_{\mathrm{CDM}}$ ). In addition, if some of the nonbaryonic dark matter is neutrinos, they will 
inevitably suppress power on small scales through freestreaming. With this in mind, let me discuss the small modifications of cold dark matter that improve its agreement with the observations.

(1) Low Hubble Constant + cold dark matter (LHC CDM). Remarkably, simply lowering the Hubble constant to around $30 \mathrm{~km} \mathrm{~s}^{-1} \mathrm{Mpc}^{-1}$ solves all the problems of cold dark matter. Recall, the critical density $\rho_{\text {crit }} \propto H_{0}^{2}$; lowering $H_{0}$ lowers the matter density and postpones matter-radiation equality, which has precisely the desired effect on the spectrum of perturbations. It has two other added benefits: it makes the expansion age of the Universe comfortably consistent with the ages of the oldest stars and raises the baryon fraction of critical density to a value that is consistent with that measured in x-ray clusters (see below). Needless to say, such a small value for the Hubble constant flies in the face of current observations; further, it illustrates the fact that the problems of cold dark matter get even worse for the larger values of $H_{0}$ that have been determined by recent observations [9].

(2) Hot + cold dark matter $(\nu \mathrm{CDM})$. Adding a small amount of hot dark matter can suppress density perturbations on small scales; of course, too much leads back to the longstanding problems of hot dark matter. The amount required is about $20 \%$, corresponding to about " $5 \mathrm{eV}$ worth of neutrinos" (i.e., one species of mass $5 \mathrm{eV}$, or two species of mass $2.5 \mathrm{eV}$, and so on). This admixture of hot dark matter rejuvenates cold dark matter provided the Hubble constant is not too large, $H_{0} \lesssim 55 \mathrm{~km} \mathrm{~s}^{-1} \mathrm{Mpc}^{-1}$.

(3) Cosmological constant + cold dark matter $(\Lambda \mathrm{CDM})$. (A cosmological constant corresponds to a uniform energy density, or vacuum energy.) Shifting $60 \%$ to $80 \%$ of the critical density to a cosmological constant lowers the matter density and has the same beneficial effect as a low Hubble constant. In fact, a Hubble constant as large as $80 \mathrm{~km} \mathrm{~s}^{-1} \mathrm{Mpc}^{-1}$ can be tolerated. In addition, the cosmological constant allows the age problem to solved even if the Hubble constant is large, addresses the fact that few measurements of the mean mass density give a value as large as the critical density (most measurements of the mass density are insensitive to a uniform component), and allows the fraction of matter in baryons to be large (see below). Not everything is rosy; cosmologists have invoked a cosmological constant twice before to solve their problems (Einstein to obtain a static universe and Bondi, Gold, and Hoyle to solve the earlier age crisis when $H_{0}$ was thought to be $250 \mathrm{~km} \mathrm{~s}^{-1} \mathrm{Mpc}^{-1}$ ). Further, particle physicists can still not explain why the energy of the vacuum is not at least 50 (if not 120) orders of magnitude larger 
than the present critical density.

(4) Extra relativistic particles + cold dark matter $(\tau \mathrm{CDM})$. The epoch of matter-radiation equality can also be delayed by raising the level of radiation. In the standard cosmology the radiation content today consists of photons + three (undetected) cosmic seas of neutrinos (corresponding to $g_{*} \simeq 3.36$ ). While we have no direct determination of the radiation beyond that in the CBR, there are at least two problems: (i) what are the additional relativistic particles?; and (ii) can additional radiation be added without upsetting the successful predictions of primordial nucleosynthesis which depend critically upon the energy density of relativistic particles. The simplest way around these problems is an unstable tau neutrino (mass anywhere between a few $\mathrm{keV}$ and a few $\mathrm{MeV}$ ) whose decays produce the radiation. This fix can tolerate a larger Hubble constant, though at the expense of more radiation.

(5) Tilted cold dark matter (TCDM). While the spectrum of density perturbations in most models of inflation is very nearly scale invariant, there are models where the deviations are significant and lead to smaller fluctuations on small scales. Further, not only do density perturbations produce CBR anisotropy, but so do the gravitational waves; if gravity waves account for a significant part of the CBR anisotropy, the level of density perturbations must be lowered. A combination of tilt and gravity waves can solve the problem of too much power on small scales, but does not seem to address the shape problem as well as the other fixes.

In evaluating these better fit models, one should keep the words of Francis Crick in mind (loosely paraphrased): A model that fits all the data at a given time is necessarily wrong, because at any given time not all the data are correct(!). $\Lambda \mathrm{CDM}$ provides an interesting example; when I discussed it in 1990, I called it the best-fit Universe, but not the best motivated and was certain it would fall by the wayside [28]. In 1995, it is still probably the best-fit model.

Let me end by defending the other point of view, namely, that to add something to cold dark matter is not unreasonable, or even as some have said, a last gasp effort to saving a dying theory. Standard cold dark matter was a starting point, similar to early calculations of big-bang nucleosynthesis. It was always appreciated that the inflationary spectrum of density perturbations was not exactly scale invariant [29] and that the Hubble constant was unlikely to be exactly $50 \mathrm{~km} \mathrm{~s}^{-1} \mathrm{Mpc}$. As the quality and quantity of data improve, it is only sensible to refine the model, just as has been done 
with big-bang nucleosynthesis. Cold dark matter seems to embody much of the "truth." The modifications suggested all seem quite reasonable (as opposed to contrived). Neutrinos exist; they are expected to have mass; there is even some experimental data that indicates they do have mass. It is still within the realm of possibility that the Hubble constant is less than $50 \mathrm{~km} \mathrm{~s}^{-1} \mathrm{Mpc}^{-1}$, and if it is as large as $70 \mathrm{~km} \mathrm{~s}^{-1} \mathrm{Mpc}^{-1}$ to $80 \mathrm{~km} \mathrm{~s}^{-1} \mathrm{Mpc}^{-1} \mathrm{a}$ cosmological constant seems inescapable based upon the age problem. There is no data that can preclude more radiation than in the standard cosmology and deviations from scale invariance were always expected.

\section{The Future}

\subsection{Testing and discriminating}

The stakes for cosmology are high: if correct, inflation/cold dark matter represents a major extension of the big bang and our understanding of the Universe, which can't help but shed light on the fundamental physics at energies of order $10^{14} \mathrm{GeV}$ or higher.

How and when we will have definitive tests of cold dark matter? Because of the large number of measurements that are being carried out and can have significant impact, I believe sooner rather than later. The list is long: CBR anisotropy; larger redshift surveys (e.g., the Sloan Digital Sky Survey will have $10^{6}$ redshifts); direct searches for the nonbaryonic in our neighborhood (e.g., axion and neutralino searches) and baryonic dark matter (microlensing); x-ray studies of galaxy clusters; the use of back-lit gas clouds (quasar absorption line systems) to study the Universe at high redshift; galactic evolution (as revealed by deep images of the sky taken by the Hubble Space Telescope and Keck 10 meter telescope); a variety of measurements of $H_{0}$ and $q_{0}$; mapping of the peculiar velocity field at large redshifts through the Sunyaev-Zel'dovich effect; dynamical estimates of the mass density (weak gravitational lensing, large-scale velocity fields, and so on); age determinations of the Universe; gravitational lensing; searches for supersymmetric particles (at accelerators) and neutrino oscillations (at accelerators, solar-neutrino detectors, and other large underground detectors); searches for high-energy neutrinos from neutralino annihilates in the sun using large underground detectors; and on and on. Consider the possible impact of a 
few specific examples.

A definitive determination that $H_{0}$ is greater than $55 \mathrm{~km} \mathrm{~s}^{-1} \mathrm{Mpc}^{-1}$ would falsify LHC CDM and $\nu$ CDM. Likewise, a definitive determination that $H_{0}$ is $75 \mathrm{~km} \mathrm{~s}^{-1} \mathrm{Mpc}^{-1}$ or larger would necessitate a cosmological constant. A flat Universe with a cosmological constant has a very different deceleration parameter than one dominated by matter, $q_{0}=-1.5 \Omega_{\Lambda}+0.5 \sim-(0.4-0.7)$ compared to $q_{0}=0.5$, and this could be settled by galaxy number counts or numbers of lensed quasars. The level of CBR anisotropy in $\tau$ CDM and LHC CDM on the $0.5^{\circ}$ scale is about $50 \%$ larger than the other models, which should be easily measurable. If neutrino-oscillation experiments were to provide evidence for a neutrino of mass $5 \mathrm{eV}$ (two of mass $2.5 \mathrm{eV}$ ) $\nu \mathrm{CDM}$ would seem almost inescapable.

A map of the CBR with $0.5^{\circ}-1^{\circ}$ resolution could separate the gravitywave from density perturbation contribution to the CBR anisotropy and provide evidence for the third robust prediction of inflation. Further, mapping CBR anisotropy on these scales or slightly smaller offers the possibility determining the geometry of the Universe (the position of the "Doppler" peak scales as $\left.0.5^{\circ} / \sqrt{\Omega_{0}}[30]\right)$.

X-ray observations of rich clusters are able to determine the ratio of hot gas (baryons) to total cluster mass (baryons + CDM) (by a wide margin, most of the baryons "seen" in cluster are in the hot gas). To be sure there are assumptions and uncertainties; the data at the moment indicate that this ratio is $0.07 h^{-3 / 2}$ [31]. If clusters provide a fair sample of the universal mix of matter, then this ratio should equal $\Omega_{B} /\left(\Omega_{B}+\Omega_{\mathrm{CDM}}\right) \simeq(0.009-$ $0.022) h^{-2} /\left(\Omega_{B}+\Omega_{\mathrm{CDM}}\right)$. Since clusters are large objects they should provide an approximately fair sample. Taking the numbers at face value, cold dark matter is consistent with the cluster gas fraction provided either: $\Omega_{B}+$ $\Omega_{\mathrm{CDM}}=1$ and $h \sim 0.3$ or $\Omega_{B}+\Omega_{\mathrm{CDM}} \sim 0.3$ and $h \sim 0.7$, favoring LHC CDM or $\Lambda$ CDM.

If cold dark matter is correct, then a significant, if not dominant, fraction of the dark halo of our galaxy should be cold dark matter (the halos of spiral galaxies are not large enough to guarantee that they represent a fair sample). Direct searches for faint stars have failed to turn up enough to account for the halo [32]. Over the past few years, microlensing has been used to search for dark stars (stars below the $0.08 M_{\odot}$ limit for hydrogen burning). Five stars in the LMC have been observed to change brightness in a way consistent with their being microlensed by dark halo objects passing along the line of sight. 
While the statistics are small, and there are uncertainties concerning the size of dark halo, these results indicate that only a small fraction (5\% to $30 \%$ ) of the dark halo is in the form of dark stars [33].

\subsection{Reconstruction}

If cold dark matter is shown to be correct, then a window to the very early Universe $\left(t \sim 10^{-34} \mathrm{sec}\right)$ will have been opened. While it is certainly premature to jump to this conclusion, I would like to illustrate one example of what one could hope to learn. As mentioned earlier, the spectra and amplitudes of the the tensor and scalar metric perturbations predicted by inflation depend upon the underlying model, to be specific, the shape of the inflationary scalar-field potential. (Inflation involves the classical evolution of a scalar field $\phi$ rolling down its potential energy curve $V(\phi)$.) If one can measure the power-law index of the scalar spectrum and the amplitudes of the scalar and tensor spectra, one can recover the value of the potential and its first two derivatives around the point on the potential where inflation took place [34]. (Measuring the power-law index of the tensor perturbations in addition, allows an important consistency check of inflation.) Reconstruction of the inflationary scalar potential would shed light both on inflation as well as physics at energies of the order of $10^{14} \mathrm{GeV}$.

\subsection{Concluding remarks}

We live in exciting times. We have a cosmological model that provides a reliable accounting of the Universe from $0.01 \mathrm{sec}$ until the present. Together with the standard model of particle physics it provides a framework for both asking deeper questions about the Universe and making sensible speculations. With inflation and cold dark matter we may be on the verge of a very significant extension of the standard cosmology. Most importantly, the data needed to test the cold dark matter theory is coming in at a rapid rate. At the very least we should soon know whether we are on the right track or if it's back to the drawing board.

This work was supported in part by the DOE (at Chicago and Fermilab) and by the NASA through grant NAGW-2381 (at Fermilab). 


\section{References}

[1] For textbooks on modern cosmology see e.g., E.W. Kolb and M.S. Turner, The Early Universe (Addison-Wesley, Redwood City, CA, 1990) or P.J.E. Peebles, Principles of Physical Cosmology (Princeton University Press, Princeton, NJ, 1993).

[2] See e.g., J. Mould et al., Astrophys. J. 383, 467 (1991).

[3] J. Mather et al., Astrophys. J. 420, 439 (1994).

[4] See e.g., M. White, D. Scott, and J. Silk, Ann. Rev. Astron. Astrophys. 32, 319 (1994).

[5] C. Copi, D.N. Schramm and M.S. Turner, Science 267, 192 (1995).

[6] See e.g., G. Efstathiou, in The Physics of the Early Universe, eds. J.A. Peacock, A.F. Heavens and A.T. Davies (Adam Higler, Bristol, 1992).

[7] See e.g., S. Weinberg, Gravitation and Cosmology (J. Wiley, New York, 1972).

[8] See e.g., H. Arp et al., Nature 346, 807 (1990).

[9] G.H. Jacoby et al., Proc. Astron. Soc. Pacific 104, 599 (1992); M. Fukugita, C.J. Hogan, and P.J.E. Peebles, Nature 366, 309 (1993); W. Freedman et al, Nature 371, 757 (1994).

[10] G. Smoot et al., Astrophys. J. 396, L1 (1992).

[11] J.P. Ostriker, Ann. Rev. Astron. Astrophys. 31, 689 (1993).

[12] F. Hoyle, G. Burbidge, and J.V. Narlikar, Astrophys. J. 410, 437 (1993); Mon. Not. R. astron. Soc. 267, 1007 (1994); Astron. Astrophys. 289, 729 (1994).

[13] S. Faber and J. Gallagher, Ann. Rev. Astron. Astrophys. 17, 135 (1979).

[14] V. Trimble, Ann. Rev. Astron. Astrophys. 25, 425 (1987). 
[15] See e.g., N. Kaiser et al., Mon. Not. R. astr. S. 252, 1 (1991); M. A. Strauss et al., Astrophys. J. 397, 395 (1992); A. Dekel, Ann. Rev. Astron. Astrophys. 32, 371 (1994).

[16] P.J.E. Peebles, Nature 327, 210 (1987); Astrophys. J. 315, L73 (1987).

[17] A. Guth, Phys. Rev. D 23, 347 (1981).

[18] M.S. Turner, Proc. Natl. Acad. Sci. (USA) 90, 4822 (1993).

[19] See e.g., N. Turok, Physica Scripta T36, 135 (1991).

[20] W. Hu and N. Sugiyama, astro-ph/9403031.

[21] P.J.E. Peebles, Astrophys. J. 432, L1 (1994).

[22] See e.g., A.D. Linde, Particle Physics and Inflationary Cosmology (Harwood, Chur, 1990), or M.S. Turner, in Proceedings of TASI-92, eds. J. Harvey and J. Polchinski (World Scientific, Singapore, 1993).

[23] Inflationary models have been constructed with $\Omega_{0}<1$; see e.g., P.J. Steinhardt, Nature 345, 47 (1990); M. Bucher et al., hepth/9411206.

[24] A. H. Guth and S.-Y. Pi, Phys. Rev. Lett. 49, 1110 (1982); S. W. Hawking, Phys. Lett. B 115, 295 (1982); A. A. Starobinskii, ibid 117, 175 (1982); J. M. Bardeen, P. J. Steinhardt, and M. S. Turner, Phys. Rev. D 28, 697 (1983).

[25] V.A. Rubakov, M. Sazhin, and A. Veryaskin, Phys. Lett. B 115, 189 (1982); R. Fabbri and M. Pollock, ibid 125, 445 (1983); A.A. Starobinskii Sov. Astron. Lett. 9, 302 (1983); L. Abbott and M. Wise, Nucl. Phys. B 244, 541 (1984).

[26] See e.g., G.R. Blumenthal et al., Nature 311, 517 (1984).

[27] A.D. Liddle and D. Lyth, Phys. Repts. 231, 1 (1993).

[28] M.S. Turner, Physica Scripta T36, 177 (1991). 
[29] P.J. Steinhardt and M.S. Turner, Phys. Rev. D 29, 2162 (1984).

[30] M. Kamionkowski et al., Astrophys. J. 426, L57 (1994).

[31] See e.g., U.G. Briel et al., Astron. Astrophys. 259, L31 (1992); S.D.M. White et al., Nature 366, 429 (1993); D.A. White and A.C. Fabian, Mon. Not. R. astron. Soc., in press (1995).

[32] See e.g., J. Bahcall et al., Astrophys. J. 435, L51 (1994) and References therein.

[33] E. Gates, G. Gyuk, and M.S. Turner, astro-ph/9411073; C. Alcock et al., astro-ph/9501091.

[34] E.J. Copeland, E.W. Kolb, A.R. Liddle, and J.E. Lidsey, Phys. Rev. Lett. 71, 219 (1993); Phys. Rev. D 48, 2529 (1993); M.S. Turner, ibid, 3502 (1993); M.S. Turner, ibid 48, 5539 (1993); L. Knox and M.S. Turner, Phys. Rev. Lett. 73, 3347 (1994); L. Knox, Phys. Rev. $D$, in press (1995). 